\title{
Electric field tuning of the band gap in four layers of graphene with different stacking order
}

\author{
Artak A. Avetisyan*a, ${ }^{*}$ Bart Partoens $^{\mathrm{b}}$, and Francois M. Peeters ${ }^{\mathrm{b}}$ \\ ${ }^{a}$ Dept. of Physics, Yerevan State Univ., A. Manoogian 1, Yerevan 0025, Armenia, \\ ${ }^{b}$ Dept. of Physics, Univ. of Antwerpen, Groenenborgerlaan 171, B-2020 Antwerpen, Belgium
}

\begin{abstract}
We investigated the effect of different stacking order of the four graphene layer system on the induced band gap when positively charged top and negatively charged back gates are applied to the system. A tight-binding approach within a self-consistent Hartree approximation is used to calculate the induced charges on the different graphene layers. We show that the electric field does not open an energy gap if the multilayer graphene system contains a trilayer part with the $A B A$ Bernal stacking.
\end{abstract}

Keywords: multilayer graphene, stacking order, induced gap, gates

\section{INTRODUCTION}

Graphene is a two-dimensional (2D) crystal of carbon atoms packed into a hexagonal lattice with a $k$-linear Dirac-like spectrum of charge carriers. The fabrication of single and multilayers of graphene stimulated the investigation of the electronic, optical, and transport properties of such graphene systems. ${ }^{1}$ Rich electronic structures and optical properties are predicted in such systems.

Ultrathin graphite films were successfully fabricated, and are very promising for nanoelectronics ${ }^{2}$ and as transparent conducting layers ${ }^{3}$ which are important for, e.g., displays and solar cells.

A serious draw-back of the use of a single layer of graphene for electronics is the observation of gapless nature of its spectrum. Theoretical and experimental investigations have shown that this difficulty can be overcome by using a perpendicular electric field applied to bilayer graphene. ${ }^{4}{ }^{5}$ This allows the opened energy gap between the valence and conduction bands to be tuned from zero to midinfrared energies. Electrical tunable energy gap systems are of interest not only from a fundamental point of view, but also for possible applications in electronics (e.g., for transistors) and photonics (i.e., wavelength tuning of a laser or an optical detector).

Recently it was shown that a tunable energy gap can also be opened in three- and four-layer graphene systems by applying a perpendicular electric field created by a single gate, and it was found that the Fermi energy was located outside the induced energy gap. ${ }^{6}$

In Ref. 7 the electronic band structure of $A B A$-stacked trilayer graphene was studied in the field of back and top gates, and the conductivity of this system was estimated. Recently, we investigated three as well as four layer graphene systems in the field of top and back gates which allows to control independently the density of electrons on the graphene layers and the Fermi energy of the system. ${ }^{8}$

*artak.avtesisyan@ua.ac.be.

Photonics and Micro- and Nano-structured Materials 2011, edited by Rafael Kh. Drampyan,

Proc. of SPIE Vol. 8414, 84140D · (c) 2012 SPIE · CCC code: 0277-786X/12/\$18 · doi: 10.1117/12.923618 
Using Raman spectroscopy measurements, the graphitic flake thickness (i.e., the number of graphene layers) can be obtained as was demonstrated in Refs. 9 and 10. In Ref. 9 a tunable three-layer graphene single-electron transistor was experimentally realized showing a transport gap near the charge neutrality point.

Multilayers of graphene can be stacked differently depending on the horizontal shift between consecutive graphene planes, leading to very different electronic and optical properties. ${ }^{11}$ The electronic low-energy band structure of the $A B C$ stacked multilayer graphene was studied within an effective mass approximation in Ref.12. It should be noted that the Bernal stacking $(A B A)$, which has hexagonal symmetry, is common and stable, but some parts of graphite can also have rhombohedral stacking $(A B C))^{13}$

In Ref. 14 we studied electric field induced band gap of multilayers of graphene with different ways of stacking. We found that the gap for trilayer graphene with the $A B C$ stacking is much larger than the one for the $A B A$ stacking. Similarly for four layers of graphene the energy gap also strongly depends on the choice of stacking and is smallest in case of Bernal stacking. We found that the effect of trigonal warping leads to considerable changes in the size of the induced electronic gap for the considered systems at intermediate and high densities of electrons.

In Ref. 15 the effect of an electric field on multilayers of graphene with different stacking was studied. Recent transport measurements on trilayers of graphene revealed the strongly stacking dependent quantum Hall effect in these systems. ${ }^{16}$ The transport properties of multilayer graphene stacks in the absence of perpendicular electric field were theoretically investigated in Ref. 17. The feasibility of identifying the stacking order of the system using transport measurments was studied. ${ }^{17}$ The Hall conductivity for the $A B A$ - and $A B C$-stacked trilayer graphene systems in perpendicular electric field is calculated in Ref 18 .

In the present paper we extend our earlier investigations and study the band structure of four layer graphene systems with the $A B B A, A B C B$ and $A B A A$ stackings in the presence of top and back gates. We use a tight-binding approach within a self-consistent Hartree approximation to calculate the induced charges on the different graphene layers.

This paper is organized as follows. The details of our tight-binding approach for four layer graphene in the presence of top and back gates with the description of the self-consistent calculation are given in section 2 . In section 3 the corresponding numerical results are discussed and section 4 summarizes our conclusions.

\section{FOUR LAYER GRAPHENE SYSTEMS IN AN EXTERNAL ELECTRIC FIELD}

In this section we consider the $A B B A$ four layer graphene system with the unit cell shown in Fig. 1a.


Figure 1. Schematic of the couplings between the different ( $A$ open and $B$ black dots) sites for four layers of graphene for: a) the $A B B A$, b) the $A B C A$ and c) the $A B C C$ stacking. 
The $A B B A$ system can be obtained from the $A B C A$ one by shifting the third layer by the in-plane lattice vector $a_{0}=2.46 \AA$ to the left. Four layers of graphene with the ABCA stacking, considered in Ref. 14 is modeled as four coupled hexagonal lattices with inequivalent sites $A_{i}$ and $B_{i}\left(i=1,2,3,4\right.$ is the layer number) with $A_{1}$ and $A_{2}, A_{3}$ and $\mathrm{B}_{2}$ as well as $\mathrm{A}_{4}$ and $\mathrm{B}_{3}$ atoms on top of each other, as shown in Fig. 1b. We use the Slonczewski-Weiss-McClure (SWMcC) parameters, i.e. $\gamma_{0}, \gamma_{1}, \gamma_{2}, \gamma_{3}, \gamma_{4}, \gamma_{5}$ of tight-binding couplings for bulk graphite. Within each layer the interaction between nearest neighbor $\mathrm{A}_{i}$ and $\mathrm{B}_{i}$ atoms is described by the parameter $\gamma_{0}$. The strong coupling between nearest layers, e.g. between $\mathrm{A}_{1}-\mathrm{A}_{2}$ atoms that lie directly above or below each other is given by $\gamma_{1}$, and the weaker nearest layer coupling by $\gamma_{3}\left(\gamma_{4}\right)$, e.g. between sites $\mathrm{B}_{1}-\mathrm{B}_{2}\left(\mathrm{~B}_{1}-\mathrm{A}_{2}\right)$. The interaction between the next nearest layers $\left(B_{1}-B_{3}\right)$ is determined by $\gamma_{2}$. The unit cell for the $A B C C$ four layer graphene is shown in Fig. 1(c) where the interaction between the $\mathrm{B}_{2}-\mathrm{A}_{4}$ atoms is determined by $\gamma_{5} \cdot{ }^{14,19}$

Using these parameters we compose the tight-binding Hamiltonian for four layer graphene with the $A B B A$ stacking, which has the form ${ }^{14}$

$$
H=\left(\begin{array}{cccc}
D_{1} & H_{12} & H_{13} & H_{14} \\
H_{21} & D_{2} & H_{23} & H_{24} \\
H_{31} & H_{32} & D_{3} & H_{34} \\
H_{41} & H_{42} & H_{43} & D_{4}
\end{array}\right)
$$

where the rows and columns are ordered according to atom $\mathrm{A}$ from layer 1, atom $\mathrm{B}$ from layer 1, atom $\mathrm{A}$ from layer 2, atom B from layer 2, etc, with the following two by two matrixes:

$$
\begin{gathered}
D_{1}=\left(\begin{array}{cc}
0 & \gamma_{0} f \\
\gamma_{0} f^{*} & 0
\end{array}\right), D_{2}=D_{3}=D_{4}=D_{1}^{\dagger}, \\
H_{12}=\left(\begin{array}{cc}
\gamma_{1} & -\gamma_{4} f^{*} \\
-\gamma_{4} f^{*} & \gamma_{3} f
\end{array}\right), H_{21}=\left(\begin{array}{cc}
\gamma_{1} & -\gamma_{4} f \\
-\gamma_{4} f & \gamma_{3} f^{*}
\end{array}\right), H_{13}=H_{31}=\left(\begin{array}{cc}
\gamma_{5} / 2 & 0 \\
0 & 0
\end{array}\right), \\
H_{23}=H_{32}=\left(\begin{array}{cc}
\gamma_{1} & -\gamma_{4} f^{*} \\
-\gamma_{4} f & \gamma_{1}
\end{array}\right), H_{34}=H_{43}^{\dagger}=\left(\begin{array}{cc}
-\gamma_{4} f & \gamma_{1} \\
\gamma_{3} f^{*} & -\gamma_{4} f
\end{array}\right), \\
H_{14}=H_{41}=\left(\begin{array}{ll}
0 & 0 \\
0 & 0
\end{array}\right), H_{42}=\left(\begin{array}{cc}
0 & 0 \\
\gamma_{5} / 2 & 0
\end{array}\right), \quad H_{24}=\left(\begin{array}{cc}
0 & \gamma_{5} / 2 \\
0 & 0
\end{array}\right),
\end{gathered}
$$

where

$$
f\left(k_{x}, k_{y}\right)=\mathrm{e}^{i k_{x} a_{0} / \sqrt{3}}+2 \mathrm{e}^{-i k_{x} a_{0} / 2 \sqrt{3}} \cos \left(k_{y} a_{0} / 2\right) .
$$


To control the density of electrons on the different graphene layers and independently the Fermi energy, a top gate with a density of negative charges $n_{t}>0$ (the electron excess density is taken positive) on it, and a back gate with a density of positive charges $n_{b}<0$ are applied to the four layer system (a schematic picture for trilayer graphene was presented in Fig. 1 of Ref. 8). As a result a total excess density $n=n_{1}+n_{2}+n_{3}+n_{4}$ is induced $\left(n=n_{t}+n_{b}\right)$, with $n_{i}$ the excess density on the $i$-th layer as counted from the top gate. In our model the top and back gate produces a uniform electric field $E_{t, b}=n_{t, b} e / 2 \varepsilon_{0} \kappa$. The graphene layers, due to the induced charges on them, create fields $E_{i}=n_{i} e / 2 \varepsilon_{0} \kappa$ with $\varepsilon_{0}$ the permittivity of vacuum, and for our numerical calculations for the dielectric constant we use the value $\kappa=2.3$ (see Ref. 4, 5) which corresponds to graphene layers on $\mathrm{SiO}_{2}$. The difference between the charge densities induced on the individual layers of graphene creates asymmetries between the first and the second, between the second and the third as well as between the third and the fourth layers, which are determined by the corresponding change in the potential energies

$$
\begin{aligned}
& \Delta_{1,2}(n)=\alpha\left(n_{2}+n_{3}+n_{4}-\left|n_{b}\right|\right) \\
& \Delta_{2,3}(n)=\alpha\left(n_{3}+n_{4}-\left|n_{b}\right|\right), \\
& \Delta_{3,4}(n)=\alpha\left(n_{4}-\left|n_{b}\right|\right)
\end{aligned}
$$

where $\alpha=e^{2} c_{0} / \varepsilon_{0} \kappa$, with $c_{0}=3.35 \AA$ the inter-layer distance.

The Hamiltonian of the $A B B A$ multilayer in the presence of the top and back gates can be obtained by adding $\Delta^{\mathrm{II}}=\Delta_{1,2}(n), \Delta^{\mathrm{III}}=\Delta_{1,2}(n)+\Delta_{2,3}(n), \Delta^{\mathrm{IV}}=\Delta_{1,2}(n)+\Delta_{2,3}(n)+\Delta_{3,4}(n)$ to the II, III, and IV layer on-site elements in Eq. (1), respectively. The tight-binding Hamiltonian operates in the space of coefficients of the tight-binding functions $c(\vec{k})=\left(c_{A_{1}}, c_{B_{1}}, c_{A_{2}}, c_{B_{2}}, c_{A_{3}}, c_{B_{3}}, c_{A_{4}}, c_{B_{4}}\right)$ where $c_{A_{i}}(\vec{k})$ and $c_{B_{i}}(\vec{k})$ are the $i$-th layer coefficients for A and B type of atoms, respectively.

By diagonalizing the Hamiltonian one can obtain the eight coefficients for fixed values of the layer asymmetries. The excess electronic densities on the individual layers are

$$
n_{i}=\frac{2}{\pi} \int d k_{x} d k_{y}\left(\left|c_{A_{i}}\right|^{2}+\left|c_{B_{i}}\right|^{2}\right) \text {; }
$$

the coefficients $C_{A_{i}}$ and $C_{B_{i}}$ depend on the band index. In order to find the redistribution of the electron density over the different layers in the valence bands one should integrate Eq. (5) over the Brillouin zone. The Fermi energy can be tuned into the opened gap, when the magnitudes of the top and back gates are equal to each other but with opposite charges on them. The case when the Fermi energy is located in the conduction or valence band was discussed in Ref. 8 for the $A B A$ stacked trilayer. Using Eqs. (1)-(5) we evaluate the energy gap $\Delta_{0}$ at the K-point and the true gap, $\tilde{\Delta}$, self-consistently for a fixed total density $n_{t}+n_{b}=n_{1}+n_{2}+n_{3}+n_{4}$ (see Refs. 4, 8).

\section{RESULTS}

In our calculations we used the parameter describing the interactions between the nearest neighbour atoms in the same layer $\gamma_{0}=3.12 \mathrm{eV}$, which leads to an in-plane velocity $v=\sqrt{3} \gamma_{0} a / 2 \hbar \approx 10^{6} \mathrm{~m} / \mathrm{s}$. For the interlayer coupling strength (i.e. between the atoms of adjacent layers which are on top of each other), we take $\gamma_{1}=0.377 \mathrm{eV}$ (see Ref. 19). Firstly we neglect all the interactions between the different atoms except $\gamma_{0}$ and $\gamma_{1}$, (i.e. we put $\gamma_{2}=\gamma_{3}=\gamma_{4}=\gamma_{5}=0$ ) that leads to a circular symmetric spectrum. Secondly, the full interaction case is studied where the interaction between the different 
atoms is expressed by the SWMcC parameters $\left(\gamma_{2}=-0.0206 \mathrm{eV}, \gamma_{3}=0.29 \mathrm{eV}, \gamma_{4}=0.12 \mathrm{eV}, \gamma_{5}=0.025 \mathrm{eV}\right)^{8,19}$ i.e. the effect of the trigonal warping is included.

Fig. 2 shows the variation of the gap $\Delta_{0}$ at the K-point (dotted curve), the true direct gap $\tilde{\Delta}$ (solid curve) and the true indirect gap $\Delta_{k k^{\prime}}$ (dot-dashed curve) with the top gate density $n_{t}$, providing the back gate density $n_{b}=-n_{t}$ ( i.e. when the Fermi energy is located in the opened gap) for four layer $A B B A$ graphene system with the full interaction. The true direct and indirect gaps are shown in Fig. 5 of Ref. 8 for the $A B A$ trilayer. It is interesting that the true gap for the $A B B A$ four layer system $\tilde{\Delta}=77.3 \mathrm{meV}$ for $n_{t}=-n_{b}=10^{13} \mathrm{~cm}^{-2}(\kappa=2.3)$ is close to the corresponding gap obtained for the $A B C C$ with $\tilde{\Delta}=70.1 \mathrm{meV}$ obtained in ${ }^{14}$. The $A B B A$ system can be realized by shifting the third layer in the $A B C A$ multilayer system (which has $\tilde{\Delta}=171 \mathrm{meV}$ at $n_{t}=-n_{b}=10^{13} \mathrm{~cm}^{-2}$ ) ${ }^{14}$ by the length of the in-plane lattice vector to the left (see Fig. 1).

We found a true direct gap for the $A B B A$ system at low densities $n_{t}=-n_{b} \approx 10^{12} \mathrm{~cm}^{-2}$, but due to the overlap between the bands at different points in k-space the indirect gap is negative as is shown in Fig. 2, i.e. we have a semi-metal for low gate densities, as in the case of the $A B C C$ multilayer graphene system. ${ }^{14}$

For the simplest case when only $\gamma_{0}, \gamma_{1} \neq 0$ the true direct gap for the $A B B A$ system $\tilde{\Delta}=\Delta_{k k^{\prime}}=89.9$ meV when the top and back gate densities $n_{t}=-n_{b}=10^{13} \mathrm{~cm}^{-2}$. We find that taking into account the full interaction between the atoms, which leads to the trigonal warping, is important for this system.

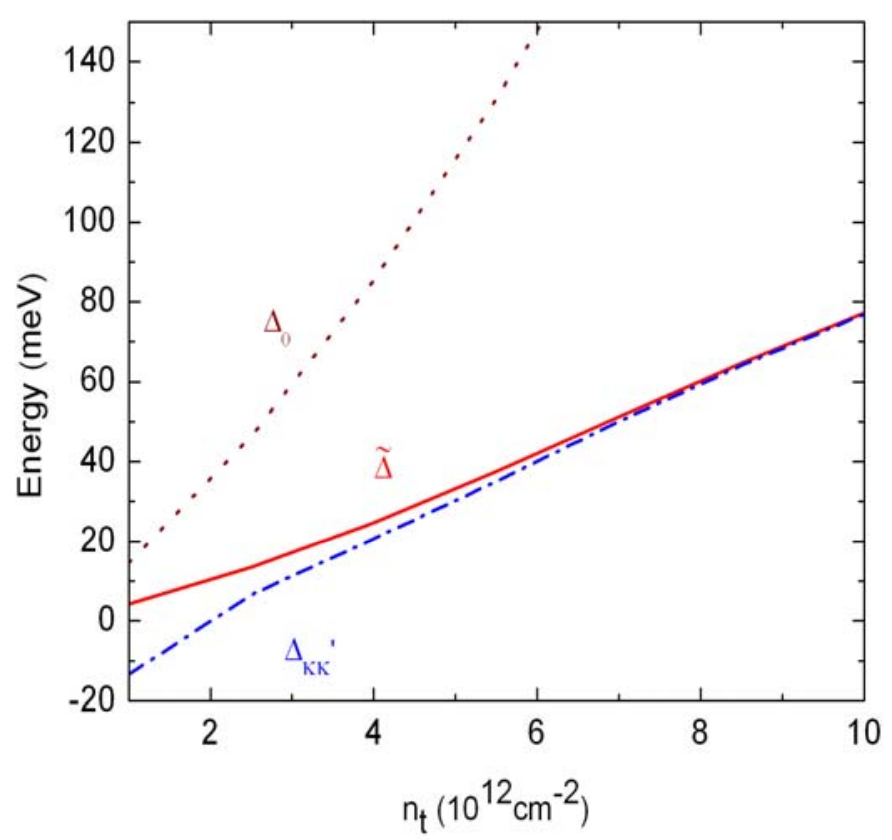

Figure 2. (Color online) The dependence of the gap $\Delta_{0}$ at the K-point (dotted curve), the true direct gap $\tilde{\Delta}$ (solid curve) and the true indirect gap $\Delta_{k k^{\prime}}$ (dot-dashed) as a function of the top gate density $n_{t}$, providing $n_{b}=-n_{t}$, for four layer graphene system $A B B A$ where we included the full interaction. 
In Fig. 3 we show the electron density as distributed over the four layers as function of the top gate electron density for the $A B B A$ system. Note that the excess densities for the $A B B A$ system on the outer layers are symmetric, and on the inner ones are almost zero.

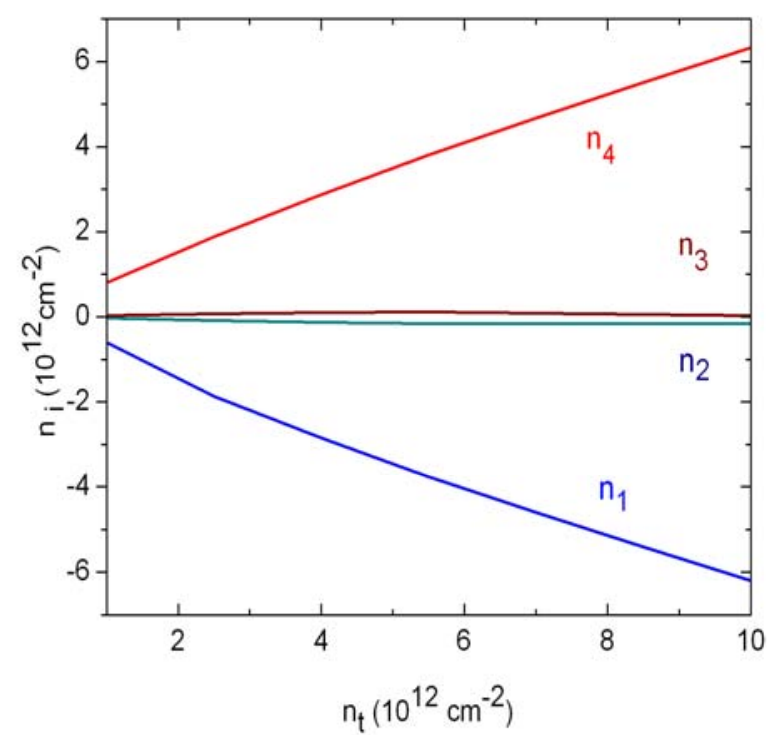

Figure3. (Color online) The layer densities $n_{i}$ for the $A B B A$ four layer graphene, when the full interaction is included, as a function of the charge density on the top gate $n_{t}$, when the magnitudes of the top and back gates are equal to each other but with opposite charges on them, i.e. $n_{b}=-n_{t}$.

Here we study also four layer graphene systems with the $A B A A$ and $A B C B$ stacking orders, which are shown in Fig. 4. We found that the electric filed can not open an energy gap in these systems.
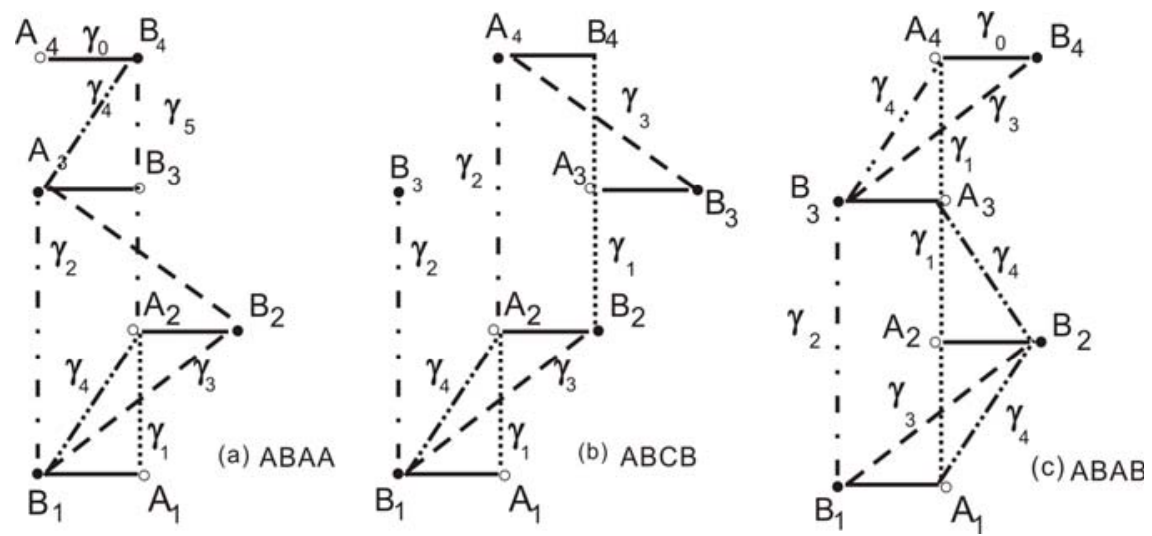

Figure 4. Schematic of the couplings between the different ( $A$ open and $B$ black dots) sites for four layers of graphene for: a) the $A B A A$, b) the $A B C B$ stacking, and c) the $A B A B$ Bernal stacking. 
It is remarkable that the $A B A A$ and $A B C B$ systems contain a trilayer with the $A B A$ Bernal stacking. A small gap in the $A B A$ trilayer as well as in the $A B A B$ four layer graphene with the Bernal stacking was obtained earlier in. ${ }^{8}$

\section{CONCLUSION}

In this paper we studied the influence of the different stacking order of four layer graphene systems on the induced band gap when positively charged top and negatively charged back gates are applied to the system, and the full interaction between graphene layers is taken into account.

Our calculations for the energy gap and the induced charges on the different graphene layers were performed using the tight-binding approach within a self-consistent Hartree approximation.

We found that a large gap (about $100 \mathrm{meV}$ ) can be opened in the $A B B A$ and $A B C C$ systems by tuning of the gate potentials. In contrast, we found that multilayer graphene which contains a trilayer with the $A B A$ Bernal stacking does not exhibit any opening of an energy gap.

\section{ACKNOWLEDGMENS}

This work was supported by the Flemish Science Foundation (FWO-Vl), and the BelgianScience Policy (IAP). One of us (A.A.A.) was supported by a fellowship from the Belgian Federal Science Policy Office (BELSPO).

\section{REFERENCES}

[1] K. S. Novoselov, A. K. Geim, S. V. Morozov, D. Jiang, M. I. Katsnelson, I. V. Grigorieva, S. V. Dubonos, and A. A. Firsov, "Two-dimensional gas of massless Dirac fermions in graphene," Nature (London) 438, 197 (2005).

[2] A. K. Geim and K. S. Novoselov, "The rise of graphene,” Nature Mater. 6, 183 (2007).

[3] P. Blake, P. D. Brimicombe, R. R. Nair, T. J. Booth, D. Jiang, F. Schedin, L. A. Ponomarenko, S. V. Morozov, H. F. Gleeson, E. W. Hill, A. K. Geim, and K. S. Novoselov, "Graphene-based liquid crystal device," Nano Lett. 8, 1704, (2008).

[4] E. McCann, “Asymmetry gap in the electronic band structure of bilayer graphene,” Phys. Rev. B 74, 161403 (R) (2006).

[5] E. V. Castro, K. S. Novoselov, S. V. Morozov, N. M. R. Peres, J. M. B. Lopes dos Santos, J. Nilsson, F. Guinea, A. K. Geim, and A. H. Castro Neto, "Biased Bilayer Graphene: Semiconductor with a Gap Tunable by the Electric Field Effect,” Phys. Rev. Lett. 99, 216802 (2007).

[6] A. A. Avetisyan, B. Partoens, and F. M. Peeters, "Electric field tuning of the band gap in graphene multilayers," Phys. Rev. B 79, 035421 (2009).

[7] M. Koshino and E. McCann, "Gate-induced interlayer asymmetry in ABA-stacked trilayer graphene," Phys. Rev. B 79, 125443 (2009).

[8] A. A. Avetisyan, B. Partoens, and F. M. Peeters, "Electric Field control of the Band Gap and Fermi Energy in Graphene Multilayers by Top and Back Gates,” Phys. Rev. B 80, 195401 (2009).

[9] J. Guttinger, C. Stampfer, F. Molitor, D. Graf, T. Ihn, and K. Ensslin,"Coulomb oscillations in three-layer graphene Nanostructures," New J. Phys. 10, 125029 (2008).

[10] D. Graf, F. Molitor, K. Ensslin, C. Stampfer, A. Jungen, C. Hierold, and L. Wirtz, "Spatially Resolved Raman Spectroscopy of Single- and Few-Layer Graphene," Nano Lett. 7, 238 (2007).

[11] S. Latil and L. Henrard, "Charge Carriers in Few-Layer Graphene Films," Phys. Rev. Lett. 97, 036803 (2006).

[12] M. Koshino and E. McCann, "Trigonal warping and Berry's phase in ABC-stacked multilayer graphene," Phys.

Rev. B 80, 165409 (2009).

[13] H. Lipson and A. R. Stokes, “The Structure of Graphite,” Proc. R. Soc. London, Ser. A 181, 101 (1942).

[14] A. A. Avetisyan, B. Partoens, and F. M. Peeters, "Stacking order dependent Electric field tuning of the band gap in graphene multilayers,” Phys. Rev. B 81, 115432 (2010).

[15] M. Koshino, "Electronic screening in graphene multilayers with ABA and ABC stacking," Phys. Rev. B 81, 125304 (2010).

[16] W. Bao, L. Jing, Y. Lee, J. Velasco Jr., P. Kratz, D. Tran, B. Standley, M. Aykol, S. B. Cronin, D. Smirnov, M. 
Koshino, E. McCann, M. Bockrath, and C.N. Lau, "Stacking-Dependent Band Gap and Quantum Transport in Trilayer Graphene," arXiv:1103.6088 (2011).

[17] Hongki Min, Parakh Jain, S. Adam, and M.D.Stiles, “ Semiclassical Boltzmann transport theory for graphene multilayer," Phys. Rev. B 83, 195117 (2011).

[18] S. Yuan, R. Roldan, and M. I. Katsnelson, "Landau level spectrum of $A B A$ - and $A B C$-stacked trilayer graphene," Phys. Rev. B 84, 125455 (2011).

[19] B. Partoens and F. M. Peeters, "From graphene to graphite: Electronic structure around the K point," Phys. Rev. B 74, 075404 (2006). 